JLAB-THY-03-01

\title{
Higher Twists in the Pion Structure Function
}

\author{
W. Melnitchouk \\ Jefferson Lab, 12000 Jefferson Avenue, Newport News, VA 23606
}

\begin{abstract}
We calculate the QCD moments of the pion structure function using DrellYan data on the quark distributions in the pion and a phenomenological model for the resonance region. The extracted higher twist corrections are found to be larger than those for the nucleon, contributing around $50 \%$ of the lowest moment at $Q^{2}=1 \mathrm{GeV}^{2}$.
\end{abstract}

Understanding the structure of the pion represents a fundamental challenge in QCD. As the lightest $q \bar{q}$ bound state, the pion presents itself as somewhat of a dichotomy: on the one hand, its anomalously small mass suggests that it should be identified with the pseudoGoldstone mode of dynamical breaking of chiral symmetry in QCD, essential for describing the long-range structure and interactions of hadrons; on the other, high energy scattering experiments reveal a rich substructure which can be efficiently described in terms of current quarks and gluons.

The duality between quark and hadron degrees of freedom reveals itself in most spectacular fashion through the phenomenon of Bloom-Gilman duality in inclusive deep-inelastic scattering. Here the inclusive $F_{2}$ structure function measured at low hadron final state mass $W$, in the region dominated by low-lying resonances, has been found in the case of the proton $[1,2]$ to follow a global scaling curve which describes high $W$ data, to which the resonance structure function averages. Furthermore, the equivalence of the averaged resonance and scaling structure functions for each prominent resonance region separately suggests that the resonance-scaling duality also exists to some extent locally.

Within QCD, the appearance of Bloom-Gilman duality for the moments of structure functions can be related through the operator product expansion to the size of high twist corrections to the scaling structure function [3]. The apparent early onset of duality for the proton structure function indicates the dominance of single-quark (leading twist) scattering even at low momentum transfers. It is not a priori clear, however, whether this is due to an overall suppression of coherent effects in inclusive scattering, or because of fortuitous cancellations of possibly large corrections. Indeed, there are some indications from models of QCD that the workings of duality may be rather different in the neutron than in the proton [4,5], or for spin-independent and spin-dependent structure functions. Given that Bloom-Gilman duality is empirically established only for baryons (specifically, the proton), while the application of theoretical models is generally more straightforward in the meson 
sector, a natural question to consider is whether, and to what extent, duality manifests itself phenomenologically for the simplest $q \bar{q}$ system in QCD - the pion.

In this note we report the first analysis of the role of resonances in the QCD moments of the pion structure function, and obtain a first estimate of the size of higher twist corrections to the scaling contribution. Similar analyses for the nucleon have been made in Refs. [6-8]. According to the operator product expansion in QCD, at large $Q^{2}$ the moments of the pion $F_{2}^{\pi}$ structure function,

$$
M_{n}\left(Q^{2}\right)=\int_{0}^{1} d x x^{n-2} F_{2}^{\pi}\left(x, Q^{2}\right)
$$

can be expanded as a power series in $1 / Q^{2}$, with coefficients given by matrix elements of local operators of a given twist $\tau$,

$$
M_{n}\left(Q^{2}\right)=\sum_{\tau=2}^{\infty} \mathcal{A}_{\tau-2}^{n}\left(\alpha_{s}\left(Q^{2}\right)\right)\left(\frac{1}{Q^{2}}\right)^{\tau-2} .
$$

Here the leading twist $\tau=2$ term $\mathcal{A}_{0}^{n}$ corresponds to free quark scattering, and is responsible for the scaling of the structure functions (modulo perturbative $\alpha_{s}\left(Q^{2}\right)$ corrections). The higher twist terms $\mathcal{A}_{\tau>2}^{n}$ represent matrix elements of operators involving both quark and gluon fields, and are suppressed by powers of $1 / Q^{2}$. The higher twist contributions reflect the strength of nonperturbative QCD effects, such as multi-parton correlations, associated with confinement.

Note that the definition of $M_{n}\left(Q^{2}\right)$ includes the elastic contribution at $W=m_{\pi}$, corresponding to $x=Q^{2} /\left(W^{2}-m_{\pi}^{2}+Q^{2}\right)=1$, which is given by the square of the elastic pion form factor, $F_{\pi}\left(Q^{2}\right)$,

$$
F_{2}^{\pi(\mathrm{el})}\left(x=1, Q^{2}\right)=2 m_{\pi} \nu\left(F_{\pi}\left(Q^{2}\right)\right)^{2} \delta\left(W^{2}-m_{\pi}^{2}\right) .
$$

Although negligible at high $Q^{2}$, the elastic contribution has been found to be important numerically at intermediate $Q^{2}$ for moments of the nucleon structure function [6-10]. In Eq. (1) we use the Cornwall-Norton moments rather than the Nachtmann moments, which are expressed in terms of the Nachtmann scaling variable, $\xi=2 x /\left(1+\sqrt{1+4 x^{2} m_{\pi}^{2} / Q^{2}}\right)$, and include effects of the target mass. Because of the small value of $m_{\pi}$, the difference between the variables $x$ and $\xi$, and therefore between the $x$-and $\xi$-moments, is negligible for the pion.

The pioneering analysis of De Rujula et al. [3] (see also Ref. [6]) showed that the size of the higher twist matrix elements directly governs the onset of quark-hadron duality. Namely, there is a region of $n$ and $Q^{2}$ in which the moments of the structure function are dominated by low mass resonances, where the higher twist contributions are neither overwhelming nor negligible. For example, even though there are large contributions from the resonance region $(W \lesssim 2 \mathrm{GeV})$ to the $n=2$ moment of the proton $F_{2}$ structure function $(\sim 70 \%$ at $Q^{2}=1 \mathrm{GeV}^{2}$ ), the higher twist effects are only of the order $10-20 \%$ at the same $Q^{2}[6]$. The question we address here is whether there is an analogous region for the pion, where the resonance contributions are important, but higher twist effects are small enough for duality to be approximately valid. 
Of course, strictly speaking the distinction between the resonance region and the deep inelastic continuum (DIS) is somewhat arbitrary, as can be illustrated in the large $N_{c}$ limit of QCD. There the final state in deep inelastic scattering from the pion is populated by infinitely narrow resonances, even in the Bjorken limit, while the structure function calculated at the parton level produces a smooth, scaling function [4,11]. Phenomenologically the spectrum of the excited states of the pion is expected to be rather smooth sufficiently above the $\rho$ mass, for $W \gtrsim 1 \mathrm{GeV}$. Contributions from the excitation of heavier mesons are not expected to be easily discernible from the DIS continuum - the $a_{1}$ meson, for instance, appears at a mass $W \sim 1.3 \mathrm{GeV}$, and has a rather broad width $(\sim 350-500 \mathrm{MeV})$.

The pion structure function has been measured in the $\pi N$ Drell-Yan process [12-14] over a large range of $x, 0.2 \lesssim x \lesssim 1$, and for $Q^{2}$ typically $\gtrsim 20 \mathrm{GeV}^{2}$. It has also been studied at HERA in semi-inclusive DIS at very low $x$ and high $W$ [15]. No data exist on $F_{2}^{\pi}$ at low $W$, however, in the region where mesonic resonances would dominate the cross section. The spectrum could in principle be reconstructed by observing low $t$ neutrons at low $W$ produced in the semi-inclusive charge-exchange reaction, ep $\rightarrow e n X$, where $t$ is the momentum transfer squared between the proton and neutron. In the absence of such data, to obtain a quantitative estimate of the importance of the resonance region, we model the pion spectrum at low $W$ in terms of the elastic and $\rho$ pole contributions, on top of the DIS continuum which is evaluated by evolving the leading twist structure function to lower $Q^{2}$. The latter can be reconstructed from parameterizations [16-18] of leading twist quark distributions in the pion obtained from global analyses of the pion Drell-Yan data. In this work we use the low $Q^{2}$ fit from Ref. [16], which gives the leading twist parton distributions in the pion for $Q^{2}>0.25 \mathrm{GeV}^{2}$, although our conclusions do not change with the use of other parameterizations $[17,18]$. For the elastic contribution we use a parameterization of the world's data [19] which interpolates smoothly between the perturbative QCD and photoproduction limits [20].

The $\rho$ contribution is described by the $\pi \rightarrow \rho$ transition form factor, $F_{\pi \rho}\left(Q^{2}\right)$, and is expected to fall as $1 / Q^{4}$ at large $Q^{2}$ (compared with $1 / Q^{2}$ for $F_{\pi}\left(Q^{2}\right)$ ). Since there is no empirical information on $F_{\pi \rho}\left(Q^{2}\right)$, we consider several models in the literature, based on a relativistic Bethe-Salpeter vertex function [21], a covariant Dyson-Schwinger approach [22], and light-cone QCD sum rules [23]. These represent a range of $\sim 100 \%$ in the magnitude of $F_{\pi \rho}\left(Q^{2}\right)$ over the region of $Q^{2}$ covered in this analysis. The calculation of Ref. [23] gives a somewhat smaller result than in Refs. [21,22], which give a similar magnitude for $F_{\pi \rho}$. The difference between these can be viewed as an estimate of the uncertainty in this contribution.

In Fig. 1 we plot the contributions to the moments of the pion structure function from the "resonance region", $M_{n}^{\text {res }}\left(Q^{2}\right)$, as a ratio to the total moment, for $n=2, \cdots, 10$. The resonance region here is defined by $W<W_{\text {res }} \equiv 1 \mathrm{GeV}$, corresponding to restricting the integral in Eq. (1) to the range $x_{\text {res }}=Q^{2} /\left(W_{\text {res }}^{2}-m_{\pi}^{2}+Q^{2}\right)$ to the elastic point at $x=1$. For the $n=2$ moment the low $W$ region contributes as much as $50 \%$ at $Q^{2}=2 \mathrm{GeV}^{2}$, decreasing to $\lesssim 1 \%$ for $Q^{2} \gtrsim 10 \mathrm{GeV}^{2}$. Higher moments, which are more sensitive to the large $x$ region, subsequently receive larger contributions from low $W$. The $n=10$ moment, for example, is virtually saturated by the resonance region at $Q^{2}=2 \mathrm{GeV}^{2}$, and still has some $40 \%$ of its strength coming from $W<1 \mathrm{GeV}$ even at $Q^{2}=10 \mathrm{GeV}^{2}$.

The large size of the resonance contributions suggests that, at a given scale $Q^{2}$, higher twist effects play a more important role in the moments of the pion structure function than 


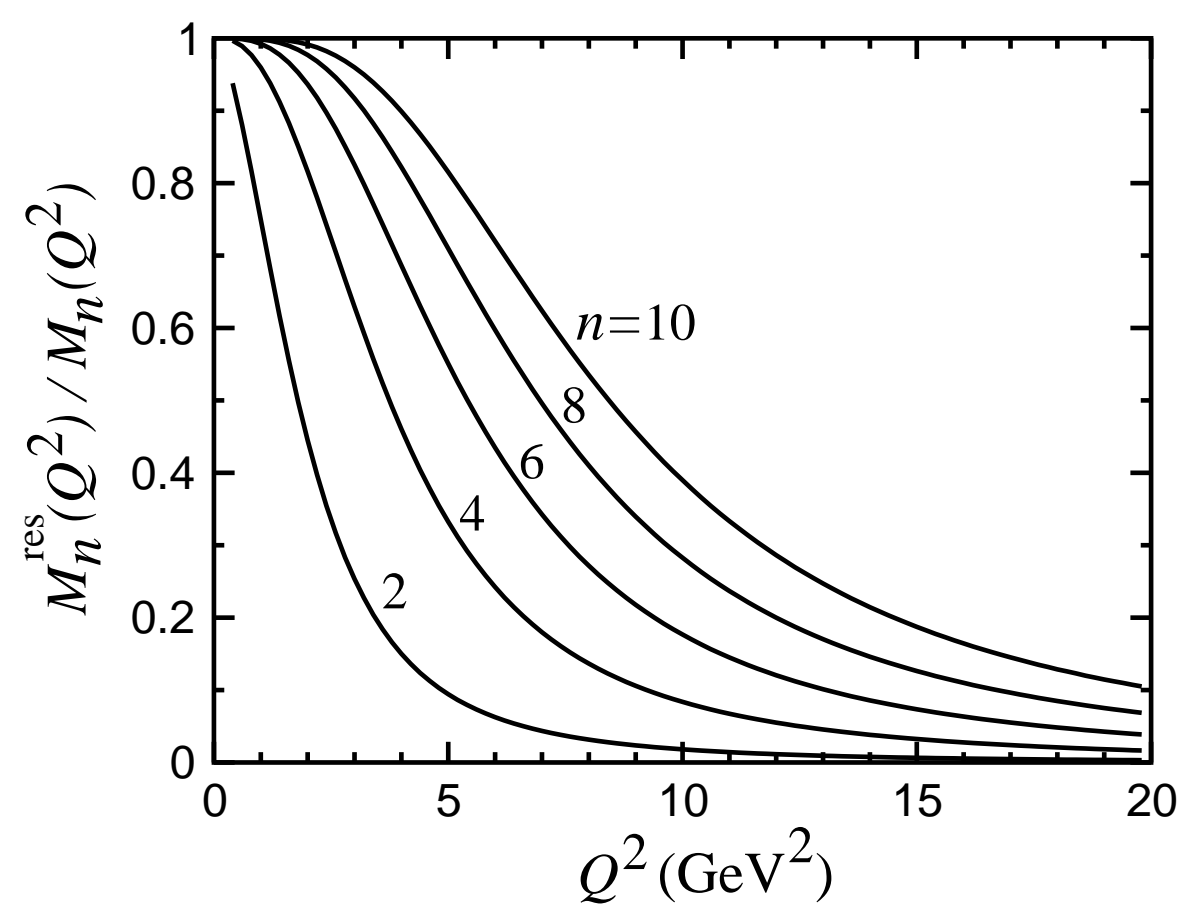

FIG. 1. Contributions to moments of the pion structure function from the resonance region, $W<W_{\text {res }}=1 \mathrm{GeV}$, relative to the total.

in the case of the nucleon. The lowest $(n=2)$ moment of $F_{2}^{\pi}$ is displayed in Fig. 2, including the leading twist and elastic contributions to $M_{2}\left(Q^{2}\right)$. The leading twist component,

$$
M_{n}^{\mathrm{LT}}\left(Q^{2}\right)=\sum_{q} e_{q}^{2} \int_{0}^{1} d x x^{n-1} q^{\pi}\left(x, Q^{2}\right),
$$

is expressed (at leading order in $\alpha_{s}\left(Q^{2}\right)$ ) in terms of the twist-2 quark distributions in the pion, $q^{\pi}\left(x, Q^{2}\right)$. The leading twist contribution is dominant at $Q^{2}>5 \mathrm{GeV}^{2}$, while the deviation of the total moment from the leading twist at lower $Q^{2}$ indicates the increasingly important role played by higher twists there. In particular, while negligible beyond $Q^{2} \approx$ $4 \mathrm{GeV}^{2}$, the elastic contribution is as large as the leading twist already at $Q^{2} \approx 1 \mathrm{GeV}^{2}$. The contribution from the $\pi \rightarrow \rho$ transition is more uncertain, and the band in Fig. 2 represents the total moment calculated using different models [21-23] of $F_{\pi \rho}\left(Q^{2}\right)$. However, while the current uncertainty in this contribution is conservatively taken to be $\sim 100 \%$, doubling this would lead to a modest increase of the band in Fig. 2. Uncertainty arising from poor knowledge of the leading twist distributions at small $x$ [16-18] is not expected to be large.

The higher twist part of the moments can be defined as the difference between the total moment $M_{n}\left(Q^{2}\right)$ and the leading twist contribution in Eq. (4), which includes a term arising from target mass corrections,

$$
M_{n}^{\mathrm{HT}}\left(Q^{2}\right)=M_{n}\left(Q^{2}\right)-M_{n}^{\mathrm{LT}}\left(Q^{2}\right)-M_{n}^{\mathrm{TM}}\left(Q^{2}\right) .
$$

Although nonperturbative effects can in principle mix higher twist with higher order effects in $\alpha_{s}$, rendering the formal separation of the two ambiguous [24] (the perturbative expansion 


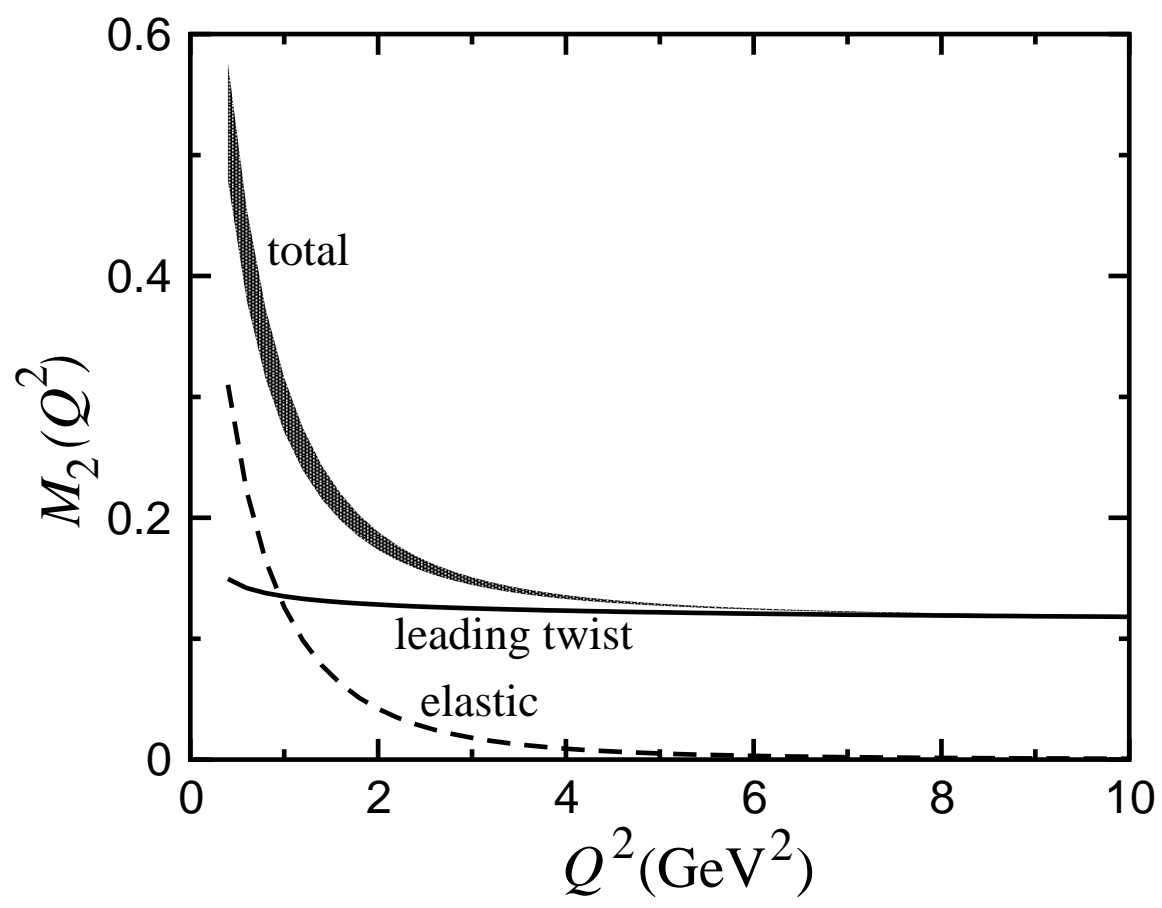

FIG. 2. Lowest $(n=2)$ moment of the pion structure function. The leading twist (solid) and elastic (dashed) contributions are shown, and the shaded region represents the total moment using different models for the $\pi \rightarrow \rho$ transition.

itself may not even be convergent), by restricting ourselves to the region of $Q^{2}$ in which the $1 / Q^{2}$ term is significantly larger than the next order correction in $\alpha_{s}$, the ambiguity in defining the higher twist terms can be neglected [6]. Because the target mass correction, $M_{n}^{\mathrm{TM}}\left(Q^{2}\right)$, which is formally of leading twist, is proportional to $m_{\pi}^{2} / Q^{2}$, its contribution will only be felt when $Q^{2} \sim m_{\pi}^{2}$, which is far from the region where the twist expansion is expected to be valid. The higher twist contribution to the $n=2$ moment is plotted in Fig. 3 as a ratio to the total moment, as a function of $Q^{2}$. The band represents an estimate of the uncertainty in the $\pi \rightarrow \rho$ transition form factor, as in Fig. 2. The higher twist contribution is as large as the leading twist at $Q^{2}=1 \mathrm{GeV}^{2}$, is around $1 / 3$ at $Q^{2}=2 \mathrm{GeV}^{2}$, and vanishes rapidly for $Q^{2} \gtrsim 5 \mathrm{GeV}^{2}$.

The higher twist contribution at $Q^{2} \sim 1 \mathrm{GeV}^{2}$ appears larger than that found in similar analyses of the proton $F_{2}[6]$ and $g_{1}$ [8] structure functions. This can be qualitatively understood in terms of the intrinsic transverse momentum of quarks in the hadron, $\left\langle k_{T}^{2}\right\rangle$, which typically sets the scale of the higher twist effects $[3,6,25,26]$. By analyzing the $x \rightarrow 1$ dependence of the measured $\mu^{+} \mu^{-}$pairs produced in $\pi N$ collisions, and the angular distribution at large $x$, the E615 Collaboration [14] indeed finds the value $\left\langle k_{T}^{2}\right\rangle=0.8 \pm 0.3 \mathrm{GeV}^{2}$ within the higher twist model of Ref. [25], which is larger than the typical quark transverse momentum in the nucleon $(\mathcal{O}(500 \mathrm{MeV}))$. The implication is that duality would therefore be expected to set in at larger $Q^{2}$ for the pion than for the nucleon.

In summary, we have evaluated moments of the pion structure function, and studied in 


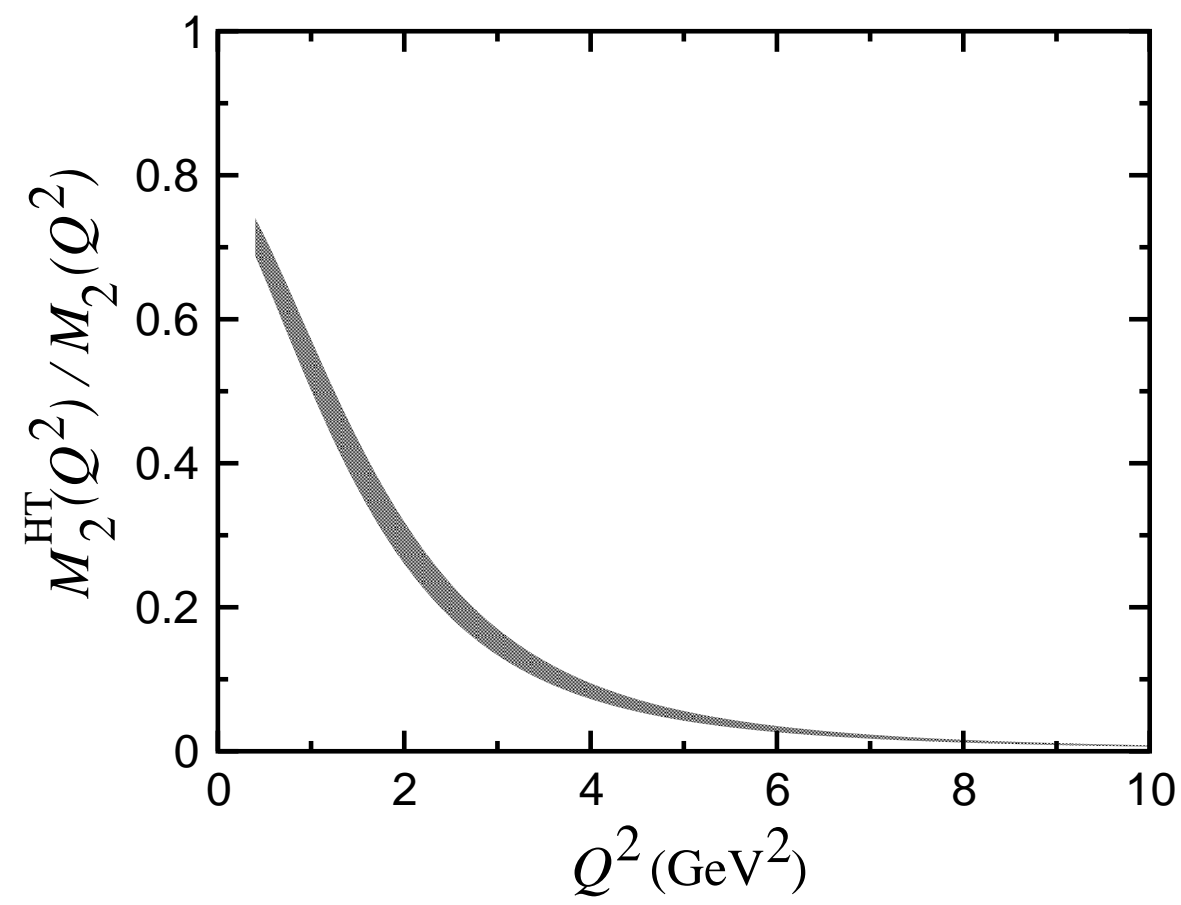

FIG. 3. Higher twist contribution to the $n=2$ moment of the pion structure function, as a ratio to the total moment. The band indicates the uncertainty due to the model dependence of the $\pi \rightarrow \rho$ transition form factor.

particular the role of the resonance region. Making the reasonable assumption that the low $W$ resonant spectrum is dominated by the elastic and $\pi \rightarrow \rho$ transitions, we have presented a first quantitative estimate of the size of higher twist contributions. For the lowest moment of $F_{2}^{\pi}$ we find that the resonance region $(W \lesssim 1 \mathrm{GeV})$ contributes $\sim 50 \%$ of the strength at $Q^{2} \approx 2 \mathrm{GeV}^{2}$, dropping to below $10 \%$ for $Q^{2} \gtrsim 5 \mathrm{GeV}^{2}$. The elastic component, while insignificant for $Q^{2} \gtrsim 3 \mathrm{GeV}^{2}$, is as large as the leading twist contribution at $Q^{2} \approx 1 \mathrm{GeV}^{2}$. The higher twist corrections to the $n=2$ moment amount to $\sim 50 \%$ at $Q^{2}=1 \mathrm{GeV}^{2}$, $\sim 30 \%$ at $Q^{2}=2 \mathrm{GeV}^{2}$, and become negligible beyond $Q^{2} \approx 6 \mathrm{GeV}^{2}$.

Uncertainties in these estimates are mainly due to the poor knowledge of the inclusive pion spectrum at low $W$, which limits the extent to which duality in the pion can be tested quantitatively. Only the elastic form factor has been accurately measured to $Q^{2} \approx 2 \mathrm{GeV}^{2}$, although at larger $Q^{2}$ it is poorly constrained. The inclusive pion spectrum can be extracted from data from the semi-inclusive charge-exchange reaction, $e p \rightarrow e n X$, at low $t$, for instance with CLAS at Jefferson Lab. This could also allow one to determine the individual exclusive channels at low $W$. In addition, a Rosenbluth separation would allow the transverse and longitudinal structure functions of the pion to be extracted. 


\section{ACKNOWLEDGMENTS}

This work was supported by the U.S. DOE contract DE-AC05-84ER40150, under which

the Southeastern Universities Research Association (SURA) operates the Thomas Jefferson National Accelerator Facility (Jefferson Lab). 


\section{REFERENCES}

[1] E. D. Bloom and F. J. Gilman, Phys. Rev. Lett. 25, 1140 (1970); Phys. Rev. D 4, 2901 (1971).

[2] I. Niculescu et al., Phys. Rev. Lett. 85, 1182 (2000); ibid 85, 1186 (2000).

[3] A. De Rujula, H. Georgi and H. D. Politzer, Annals Phys. 103, 315 (1977).

[4] N. Isgur, S. Jeschonnek, W. Melnitchouk and J. W. Van Orden, Phys. Rev. D 64, 054005 (2001).

[5] F. E. Close and N. Isgur, Phys. Lett. B 509, 81 (2001).

[6] X. Ji and P. Unrau, Phys. Rev. D 52, 72 (1995).

[7] C. S. Armstrong et al., Phys. Rev. D 63, 094008 (2001).

[8] X. Ji and P. Unrau, Phys. Lett. B 333, 228 (1994); X. Ji and W. Melnitchouk, Phys. Rev. D 56, 1 (1997).

[9] R. Ent, C. E. Keppel and I. Niculescu, Phys. Rev. D 62, 073008 (2000).

[10] W. Melnitchouk, Phys. Rev. Lett. 86, 35 (2001); Nucl. Phys. A 680, 52 (2000).

[11] M. B. Einhorn, Phys. Rev. D 14, 3451 (1976).

[12] J. Badier et al., Z. Phys. C 18, 281 (1983).

[13] B. Betev et al., Z. Phys. C 28, 15 (1985).

[14] J. S. Conway et al., Phys. Rev. D 39, 92 (1989).

[15] G. Levman, J. Phys. G 28, 1079 (2002).

[16] M. Gluck, E. Reya and A. Vogt, Z. Phys. C 53, 651 (1992).

[17] P. J. Sutton, A. D. Martin, R. G. Roberts and W. J. Stirling, Phys. Rev. D 45, 2349 (1992).

[18] M. Gluck, E. Reya and M. Stratmann, Eur. Phys. J. C 2, 159 (1998).

[19] H. P. Blok, G. M. Huber and D. J. Mack, nucl-ex/0208011.

[20] W. Melnitchouk, hep-ph/0208258, to appear in Eur. J. Phys. A.

[21] H. Ito and F. Gross, Phys. Rev. Lett. 71, 2555 (1993).

[22] P. Maris and P. C. Tandy, Phys. Rev. C 65, 045211 (2002).

[23] A. Khodjamirian, Eur. Phys. J. C 6, 477 (1999).

[24] A. H. Mueller, Phys. Lett. B 308, 355 (1993).

[25] E. L. Berger and S. J. Brodsky, Phys. Rev. Lett. 42, 940 (1979).

[26] J. F. Gunion, P. Nason and R. Blankenbecler, Phys. Rev. D 29, 2491 (1984). 